\title{
Heredity in sarcoidosis: a registry-based twin study
}

\author{
A Sverrild, ${ }^{1}$ V Backer, ${ }^{1} \mathrm{~K} O \mathrm{Kyvik},{ }^{2} \mathrm{~J}$ Kaprio, ${ }^{3} \mathrm{~N}$ Milman, ${ }^{4}$ C B Svendsen, ${ }^{5}$ S F Thomsen
}

${ }^{1}$ Department of Respiratory

Medicine, Bispebjerg University

Hospital, Copenhagen, Denmark;

${ }^{2}$ Institute of Regional Health

Research Services and The

Danish Twin Registry, University

of Southern Denmark, Odense,

Denmark: ${ }^{3}$ The Finnish Twin

Cohort Study, Department of

Public Health, University of

Helsinki, Helsinki, Finland and

Department of Mental Health

and Alcohol Research, National

Public Health Institute, Helsinki,

Finland; ${ }^{4}$ The Heart Centre,

Department of Lung

Transplantation, Rigshospitalet,

Copenhagen, Denmark;

${ }^{5}$ Department of Bacteriology,

Mycology and Parasitology,

Statens Serum Institut,

Copenhagen, Denmark

Correspondence to:

Dr A Sverrild, Department of

Respiratory Medicine, Bispebjerg

Hospital, DK-2400 Copenhagen

NV, Denmark; asgersverrild@

stud.ku.dk

Received 28 November 2007

Accepted 23 April 2008

Published Online First

5 June 2008

\section{ABSTRACT}

Background: Sarcoidosis is a multiorgan granulomatous inflammatory disease of unknown aetiology. Familial clustering of cases and ethnic variation in the epidemiology suggests a genetic influence on susceptibility to the disease. This paper reports twin concordance and heritability estimates of sarcoidosis in order to assess the overall contribution of genetic factors to the disease susceptibility.

Methods: Monozygotic and dizygotic twins enrolled in the Danish and the Finnish population-based national twin cohorts (61 662 pairs in total) were linked to diagnostic information on sarcoidosis obtained from the Danish National Patient Registry or the Social Insurance Institution, Finland registry of reimbursed medication using the 8th and 10th editions of the International Classification of Diseases. The Fisher exact test was used to compare probandwise concordance rates in different zygosity groups. Heritability was estimated based on a multifactorial threshold liability model.

Results: A total of 210 twin pairs with at least one proband with a diagnosis of sarcoidosis were identified. The probandwise concordance rate was higher in monozygotic than in dizygotic twins (0.148 vs 0.012 ). Compared with the general population there was an 80fold increased risk of developing sarcoidosis in co-twins of affected monozygotic brothers or sisters. The increased risk in dizygotic twins was only 7-fold. Aetiological model fitting gave a heritability of sarcoidosis of $0.66(95 \% \mathrm{Cl}$ 0.45 to 0.80$)$.

Conclusions: This study suggests that genetic factors play an important role in the susceptibility to sarcoidosis. This result should encourage the search for molecular genetic markers of susceptibility to the disease.

Sarcoidosis is a rare multiorgan immune-mediated disease of unknown aetiology characterised by the formation of non-caseating granulomas. Ninety percent of patients are affected in the lungs or intrathoracic lymph nodes, but large differences in organ involvement are found between ethnic groups and in different parts of the world. ${ }^{1-4}$

The prognosis and course of the disease depends on the severity and phenotypic characteristics. Overall, 30\% undergo spontaneous remission while another $30 \%$ progress with chronic manifestations. In addition, a significant number of asymptomatic cases with sarcoidosis have been identified in mass screenings using chest radiographs. ${ }^{2}{ }^{3}$ Mortality rates in patients with sarcoidosis have been reported to be $1-6 \% .^{235}$

The occurrence of sarcoidosis in the general population remains difficult to establish due to the wide spectrum of phenotypes, differing diagnostic criteria and the variable study designs. ${ }^{126}$ Incidence rates in Denmark and Finland are 7.2 and 11.4 per 100000 person-years, respectively. ${ }^{67}$
Prevalence and incidence are dependent on age, sex and ethnicity. ${ }^{1} 2$ 8-10

The present understanding of the pathogenesis of the disease is that sarcoidosis is triggered by an abnormal immune response to an environmental agent in a genetically predisposed individual. ${ }^{1}$ Genetic factors are considered to contribute to the development of sarcoidosis for two main reasons ${ }^{12610}$; (1) epidemiological studies have identified ethnicity as an important risk factor; and (2) familial clustering of cases has been observed frequently over the last decades. A previous study has suggested an up to 5.7-fold increased risk of developing sarcoidosis in siblings of affected individuals. ${ }^{9}$ However, the fact that families do not only share genes but also environment makes it difficult to determine the relative contribution of genetic versus environmental factors to the susceptibility to the disease.

Susceptibility loci have been mapped to the human leucocyte antigen (HLA) gene complex located on the short arm of chromosome 6, whereas a number of non-HLA candidate genes map to other locations in the genome. ${ }^{10-12}$ This suggests the influence of a large number of interacting genes. The specific gene findings, however, account for only a small fraction of the variance in risk to the disease.

This study is the first to use the classical twin method to systematically describe the occurrence of sarcoidosis in Danish and Finnish twins in order to address the overall contribution of genetic factors to the susceptibility to the disease.

\section{METHODS}

The twin method is a classic approach to study the heredity of a disease. Besides sharing their upbringing and early environment, monozygotic twins have all their genes in common whereas dizygotic twins only share genes like normal siblings. Therefore, if a disease is more correlated in monozygotic than in dizygotic twins, it provides evidence that genetic factors contribute to the aetiology of the disease. ${ }^{13}$

\section{Danish and Finnish twin cohorts}

The Scandinavian countries have some of the oldest and most well characterised twin registries in the world, making collaboration between countries advantageous when studying rare diseases such as sarcoidosis. ${ }^{14} 15$ The Danish study population included all twins enrolled in the nationwide Danish Twin Registry who were alive at some point between 1977 and 2004. ${ }^{16}$ This comprises a total number of 60756 pairs. The Old Finnish Twin Cohort consists of 13888 pairs of known zygosity comprising all same-sex twins born before 1958 with both twins alive in 1975. In both registries zygosity has been determined using questions of similarity and mistaken identity. ${ }^{17}$ This 
method is widely accepted and assigns zygosity correctly in more than $96 \%$ of cases. $^{18}$

\section{Case identification}

Diagnostic criteria were limited to codes 135.99 and D86.0-D86.9 of the 8th and 10th editions of the International Classification of Diseases. Information on sarcoidosis in the Danish cohort was gathered from the Danish National Patient Registry (DNPR) at the Danish Board of Health on all twins alive between 1977 and 2004. The DNPR contains details on all hospital admissions in Denmark including information on all diagnostic coding. Cases in the Finnish cohort included all twins who had applied for medical reimbursement associated with a diagnosis of sarcoidosis in the period from 1976 to 2004 (which included 11186 twin pairs of working age and not on disability pension in 1975). The Social Insurance Institution, Finland, grants the right to full reimbursement based on a medical certificate from an appropriate medical specialist or hospital unit.

\section{Statistical analysis}

The Fisher exact test was used to compare probandwise concordance rates in different zygosity groups. The probandwise concordance rate denotes the probability that one twin has the disease given the co-twin is affected, and is estimated as two times the number of concordant affected pairs (both twins are affected) divided by two times the number of concordant affected pairs plus the number of discordant pairs (one twin is affected). ${ }^{19}$

Recurrence risk ratios were calculated by dividing the probandwise concordance rate by the prevalence rate, denoting the increased risk of disease relative to the general population.

The heritability, which is the proportion of variation in susceptibility ascribable to genetic differences, was estimated from the number of unaffected, discordant and concordant pairs. This was based on a multifactorial threshold liability model and computed according to the methods described by Neale and Cardon. ${ }^{20}$

\section{RESULTS}

Of 61662 twin pairs with known zygosity, a total of 210 twin pairs were identified with at least one diagnosed proband. The distribution of subjects is shown in table 1. The Danish and Finnish cohorts did not differ significantly with regard to the prevalence in the two groups of twins, nor did the prevalence in monozygotic and dizygotic twins compared in each cohort. Pooled analysis of the data showed that the probandwise concordance rate was higher in monozygotic twin pairs than in dizygotic twin pairs (0.148 vs $0.012, \mathrm{p}=0.012)$.
Moreover, a co-twin of an affected identical twin has an 80fold increased risk of getting sarcoidosis compared with the general population, whereas the risk was increased only 7-fold in a co-twin of an affected dizygotic twin.

We found that an aetiological model including genetic and non-shared environmental effects fitted the data best with a heritability estimate of 0.66 (95\% confidence interval (CI) 0.45 to 0.80$)$. The proportion of variance explained by non-shared environmental factors was 0.34 (95\% CI 0.20 to 0.55$)$.

\section{DISCUSSION}

Sarcoidosis has been studied in affected families to reveal any possible genetic influence on susceptibility. Since families share not only genes but also environment, aggregation of cases may be due either to inherited factors or environmental exposures. The increased relative risk of 5.8 in siblings found in the ACCESS study ${ }^{9}$ concurs well with our equivalent finding in dizygotic twins. However, no studies have partitioned observed familial clustering into genetic and environmental components of variance.

The significantly higher concordance rate in monozygotic twins in this study shows that genetic factors appear to play a substantial role in the susceptibility to sarcoidosis. The heritability reflects the difference between monozygotic and dizygotic twin pairs, which tells us that genetic factors account for two-thirds of the variation in the susceptibility to the disease whereas environmental factors account for one-third.

Since monozygotic twins are mostly discordant for the disease, we need to emphasise the importance of additional environmental risk factors. The heritability estimate does not reflect the overall risk of getting the disease, merely the variance between the twins. The genetic influence on the overall risk of developing sarcoidosis is reflected in the 7-fold versus 80-fold increased risk in dizygotic and monozygotic twins, respectively. Aggregation of disease in twins has been described in the medical literature previously, ${ }^{21}$ but not with the requisite statistical power to identify any difference or heritability. It now seems evident that a considerable component of the disease accumulation is due to genetic influences on susceptibility.

In spite of the large sample size, we were still left with relatively few cases to analyse owing to the rarity of the disease. By pooling the two cohorts we benefited from a larger number of cases, particularly concordant twin pairs, which also seemed logical since the prevalence was similar in the two cohorts. The Finnish data built on reimbursement inclusion criteria which do not include patients with no need for treatment. An imbalance of more severe cases is therefore to be expected. There was no available information on the phenotypic distribution of the

Table 1 Occurrence and resemblance between twins for sarcoidosis in 61662 Danish and Finnish twin pairs

\begin{tabular}{cccccc}
\hline Zygosity & Pairs (n) & Prevalence, $\mathbf{n}(\%)$ & $\begin{array}{l}\text { Discordant } \\
\text { pairs }(\mathbf{n})\end{array}$ & $\begin{array}{l}\text { Concordant } \\
\text { pairs }(\mathbf{n})\end{array}$ & $\mathbf{C}_{\mathbf{P r}}$ \\
\hline $\begin{array}{c}\text { Denmark } \\
\text { MZ }\end{array}$ & 11085 & $41(0.185)$ & 37 & 2 & 0.098 \\
DZ & 39391 & $129(0.164)$ & 127 & 1 & 0.016 \\
Finland & & $13(0.185)$ & 9 & 2 & 0.038 \\
MZ & 3519 & $32(0.209)$ & 32 & 0 & 0 \\
DZ & 7667 & $54(0.185)$ & 46 & 4 & 0.148 \\
All MZ & 14604 & $161(0.171)$ & 159 & 1 & 0.012 \\
All DZ & 47058 & $215(0.174)$ & 205 & 5 & 0.030 \\
Total & 61662 & & & & \\
\hline
\end{tabular}

$\mathrm{C}_{\mathrm{Pr}}$, probandwise concordance rate. 
cases, so it was not possible to distinguish between genetic influences on the overall susceptibility and genetic influence on varying phenotypes as has been suggested by others. ${ }^{10} 11$

Our results support the notion of sarcoidosis as a complex disease triggered by a combination of environmental and genetic risk factors. To get further insight into the interactions causing the disease, specific genetic, environmental and infectious risk factors need to be investigated.

Acknowledgements: The authors acknowledge the Social Insurance Institution, Finland for permission to access the medication reimbursement data and financial support.

Funding: The Finnish Twin Cohort is supported by the Academy of Finland Centre of Excellence in Complex Disease Genetics.

Competing interests: None.

\section{REFERENCES}

1. ATS/ERS/WASOG. Statement on sarcoidosis. Eur Respir J 1999;14:735-7.

2. Thomeer M, Desmedts M, Wuyts W. Epidemiology of sarcoidosis. Eur Respir Monogr 2005; 32:13-22.

3. Lynch JP 3rd, Ma YL, Koss NM, et al. Pulmonary sarcoidosis. Semin Respir Crit Care Med 2007;28: 53-74.

4. Baughman RP, Teirstein AS, Judson MA, et al. Clinical characteristics of patients in a case control study of sarcoidosis. Am J Respir Crit Care Med 2001;164:1885-9.

5. Byg KE, Milman N, Hansen S. Sarcoidosis in Denmark 1980-1994. A registry-based incidence study comprising 5536 patients. Sarcoidosis Vasc Diffuse Lung Dis 2003:20:46-52.
6. Newman LS. Aetiologies of sarcoidosis. Eur Respir Monogr 2005;32:23-48.

7. Pietinalho A, Ohmichi M, Hiraga $Y$, et al. The mode of presentation of sarcoidosis in Finland and Hokkaido, Japan. A comparative analysis of 571 Finnish and 686 Japanese patients. Sarcoidosis Vasc Diffuse Lung Dis 1996;13:159-66.

8. Rybicki BA, Major M, Popovich J Jr, et al. Racial differences in sarcoidosis incidence: a 5-year study in a health maintenance organization. Am J Epidemiol 1997; 145:234-41.

9. Rybicki BA, lannuzzi MC, Fredrick MM, et al. Familial aggregation of sarcoidosis. A case-control etiologic study of sarcoidosis (ACCESS). Am J Respir Crit Care Med 2001;164:2085-91.

10. Luisetti M, Beretta A, Casali L. Genetic aspects in sarcoidosis. Eur Respir J 2000;16:768-80.

11. du Bois RM, Beirne PA, Anevlavis SE. Genetics. Eur Respir Monogr 2005;32:64-81

12. Iannuzzi MC, Rybicki BA. Genetics of sarcoidosis. Proc Am Thorac Soc 2007;4:108-16

13. Hawkes CH. Twin studies in medicine: what do they tell us? O J Med 1997;90:311-21.

14. Skytthe A, Kyvik K, Bathum L, et al. The Danish Twin Registry in the new millennium. Twin Res Hum Genet 2006;9:763-71.

15. Kaprio J, Koskenvuo M. Genetic and environmental factors in complex diseases: The Older Finnish Twin Cohort. Twin Res 2002;5:358-65.

16. Skytthe A, Kyvik K, Holm NV, et al. The Danish Twin Registry: 127 birth cohorts of twins. Twin Res 2002;5:352-7.

17. Sarna S, Kaprio J, Sistonen $P$, et al. Diagnosis of twin zygosity by mailed questionnaire. Hum Hered 1978;28:241-54.

18. Christiansen L, Frederiksen H, Schousboe K, et al. Age- and sex-differences in the validity of questionnaire-based zygosity in twins. Twin Res 2003;6:275-8.

19. McGue M. When assessing twin concordance, use the probandwise not the pairwise rate. Schizophr Bull 1992;18:171-6.

20. Neale MC, Cardon LR. Methodology for studies of twins and families. NATO ASI Series. Dordrecht, Netherlands: Kluwer Academic, 1990.

21. McGrath DS, Daniil Z, Foley P, et al. Epidemiology of familial sarcoidosis in the UK Thorax 2000:55:751-4.

\section{Lung alert}

\section{Mechanism of secondary bacterial infection following viral pulmonary infection (the viral-bacterial synergy)}

This paper examines the mechanism causing secondary bacterial infection which often occurs after pulmonary viral infection. C57BL/6 mice were infected intranasally with influenza virus strain A/PR8/34(H1N1). By day 12 there was immune-mediated viral clearance; however, the mice showed a high susceptibility to pneumococcal infection during this recovery stage.

Normal initial bacterial clearance requires innate immunity with bacterial phagocytosis by resident alveolar macrophages. The phagocytic activity of CD11c+ alveolar macrophages in bronchoalveolar lavage fluid was lower in virus-infected mice than in virus-naïve mice. To determine the influence of interferon $\gamma(\operatorname{IFN} \gamma)$, the naïve mice were inoculated with exogenous IFN $\gamma$ which inhibited alveolar macrophage-mediated phagocytosis of pneumococci both in vitro and in vivo. There was also suppressed surface expression of the class A scavenger receptor MARCO which is responsible for phagocytosis of pneumococci by alveolar macrophages. Thirdly, IFN $\gamma$ increased the surface expression of MHC class II antigen. Thus, IFN $\gamma$ inhibits bacterial phagocytosis and suppresses innate defence against pneumococcal infection. In vivo treatment with the IFN $\gamma$-specific antibody XMG1.2 had little effect on the course of viral infection but prevented increased susceptibility to pneumococcal infection by improved macrophage expression of inflammatory cytokines and preventing pre-regulation of MHC class II expression.

This study focuses on the early stages of pneumococcal infection and the crucial role of alveolar macrophages, the mechanisms of the synergy between influenza virus and Streptococcus pneumoniae and the inhibitory action of IFN $\gamma$. There might also be a future therapeutic approach of IFN $\gamma$ neutralising antibodies for preventing secondary bacterial infections.

- Sun K, Metzger DW. Inhibition of pulmonary antibacterial defense by interferon- $\gamma$ during recovery from influenza infection. Nat Med 2008:14:558-64

\section{Bhagi Jayaraman}

Correspondence to: Dr B Jayaraman, Specialist Registrar, South East Thames Deanery, London, UK; bhagi.jayaraman@ googlemail.com 